\title{
Transport through dirty interfaces
}

\author{
Kees M. Schep* and Gerrit E. W. Bauer \\ Faculty of Applied Physics and Delft Institute of Microelectronics and Submicrontechnology, \\ Delft University of Technology, Lorentzweg 1, 2628 CJ Delft, The Netherlands
}

(Received 2 June 1997)

\begin{abstract}
The transport properties of a single dirty interface are calculated starting from the Schrödinger equation. The disordered scattering potential is modeled by a high density of short-range scatterers, randomly distributed in a plane perpendicular to the direction of transport. The distribution function of transmission matrix eigenvalues is shown to be universal in the sense that it scales with a single parameter, the conductance, and does not depend on the dimension or the precise values of the microscopic parameters. It differs, however, from the well-known universal distribution for diffusive bulk conductors. These general results are supported by analytical and numerical calculations of the conductance and the angular dependence of the transmission and reflection probabilities as a function of the microscopic parameters. The conductance fluctuations are nonuniversal and a localization transition does not occur. [S0163-1829(97)00348-2]
\end{abstract}

\section{INTRODUCTION}

Most studies of phase-coherent electrical transport in disordered metals are restricted to bulk conductors in the weak scattering regime. Several interesting phenomena are observed in these systems. ${ }^{1}$ For example, when the conductance is smaller than the conductance quantum $e^{2} / h$, the electron wave functions are localized, causing the conductance to drop exponentially with the sample length. Another fascinating phenomenon is the universality of some transport properties that do not depend on the precise values of the microscopic parameters. The best known example of this is provided by the universal conductance fluctuations: ${ }^{2}$ The sample-to-sample variations of the conductance are of the order of $e^{2} / h$, independent of the sample shape and size, the degree of disorder, and the dimensionality.

In this paper we consider transport through dirty interfaces. The term "dirty" implies that the scattering is due to a random potential and that the conductance $g$ (in units $e^{2} / h$ ) is sufficiently smaller than the number of conducting channels $N(g \ll N)$. For an interface the scattering region with length $L$ (the "thickness" of the interface) is supposed to be sufficiently shorter than the Fermi wavelength $\lambda_{F}$. In contrast, the calculations for diffusive bulk conductors are in the regime $L \gg \lambda_{F}$. The aim of this work is to investigate to what extent the transport through strongly disordered interfaces is different from or similar to diffusive transport in the bulk. Besides the purely theoretical interest, our study of dirty interfaces is motivated by experiments on transport through metallic interfaces in magnetic multilayers exhibiting giant magnetoresistance. ${ }^{3,4}$ These interfaces strongly scatter electrons in a region with a length comparable to or smaller than $\lambda_{F} \cdot{ }^{5}$ Experiments of transport through a narrow disordered region in a two-dimensional electron gas are in progress. ${ }^{6}$ With some modifications the present calculations are also applicable to other scattering problems, such as the transparency of a thin, yet strongly diffusing medium to light.

Many properties of a disordered conductor can be obtained directly from the distribution function (or density) of transmission matrix eigenvalues. The transmission matrix $\mathbf{t}_{p} \mathbf{t}_{p}^{\dagger}$ is the product of the transmission amplitude matrix $\mathbf{t}_{p}$ and its Hermitian conjugate. The matrix $\mathbf{t}_{p}$ collects the transmission amplitudes of the propagating $(p)$ states on the lefthand side of a scattering region to those on the right-hand side. All propagating states are normalized to carry unit flux in the direction of transport. The distribution function $P(T)$ of the eigenvalues $T_{n}$ of the matrix $\mathbf{t}_{p} \mathbf{t}_{p}^{\dagger}$ is defined as

$$
P(T) \equiv\left\langle\sum_{n} \delta\left(T-T_{n}\right)\right\rangle
$$

where the angular brackets indicate averaging over all possible realizations of disorder in a given Hamiltonian. This distribution function can be used (see, for example, Ref. 7) to express the average value of any property $a$ that is described by a linear statistic $a(T)$ as

$$
\langle a\rangle=\left\langle\sum_{n} a\left(T_{n}\right)\right\rangle=\int d T a(T) P(T) .
$$

The conductance $g$, for example, is related to the transmission matrix by the Landauer formula

$$
g=\operatorname{Trt}_{p} \mathbf{t}_{p}^{\dagger}=\sum_{n} T_{n}
$$

and is thus described by the linear statistic $g(T)=T$. Similarly, the shot-noise power $p$ (in units $2 e|V| e^{2} / h$, with $V$ the applied voltage) is described by the linear statistic $p(T)$ $=T(1-T){ }^{8}$ Also for a normal metal/superconductor (NS) junction the conductance $g_{\mathrm{NS}}$ and the shot-noise power $p_{\mathrm{NS}}$ can be expressed in terms of the transmission matrix eigenvalues of the normal-metal region by the linear statistics $g_{\mathrm{NS}}(T)=2 T^{2} /(2-T)^{2} \quad\left(\right.$ Ref. 9) and $p_{\mathrm{NS}}(T)=16 T^{2}(1$ $-T) /(2-T)^{4},{ }^{10}$ respectively.

For disordered bulk conductors in the metallic regime (where $1 \ll g \ll N$ ) the distribution function of transmission matrix eigenvalues has been shown to be universal: ${ }^{11-13}$ 


$$
P(T)=\frac{\langle g\rangle}{2} \frac{1}{T \sqrt{1-T}} \quad \text { for } \cosh ^{-2}\left(\frac{N}{\langle g\rangle}\right)<T<1
$$

and $P(T)=0$ otherwise. The cutoff at small $T$ is such that $\int_{0}^{1} d T P(T)=N$, which for $N \gg g$ does not affect the averages of the first- and higher-order moments of $T$. Equation (4) does not depend on the shape of the conductor or the spatial resistivity distribution. The distribution function is bimodal: Most eigenvalues are either close to 1 ("'open" channels) or close to 0 ("closed" channels). This is in contrast to the naive notion that all eigenvalues should be much smaller than 1 for $g \ll N$. It follows directly ${ }^{14}$ from Eq. (4) that $\langle p\rangle /\langle g\rangle=1 / 3$, which is only one-third of the classical value for a Poisson process with $T_{n} \ll 1$. The universality of $P(T)$ has its limits. Either close to the localization regime ${ }^{12}(g$ $\approx 1$ ) or close to the ballistic regime ${ }^{15}(g \leqq N)$ Eq. (4) is no longer valid. Even in the metallic regime where $1 \ll g \ll N$ the universality can be broken by extended defects, such as tunnel barriers, grain boundaries, or interfaces. ${ }^{13}$

In this paper a microscopic calculation is presented in which the scattering potential at the interface is modeled by scatterers with short-range potentials that are randomly distributed on a plane. The main result is a universal distribution function of transmission matrix eigenvalues for a single dirty interface that differs from Eq. (4) for bulk systems. In other words, dirty interfaces $\left(L \ll \lambda_{F}\right)$ belong to a universality class ${ }^{16}$ different from disordered bulk conductors $(L$ $\gg \lambda_{F}$ ). In addition, a localization transition does not exist, the conductance fluctuations are nonuniversal, and the angular dependences of the transmission and reflection probabilities differ from those of bulk conductors. A short account of part of this work was given in Ref. 17.

The paper is organized as follows. In Sec. II the model is introduced and the scattering properties are expressed in terms of the microscopic parameters. In Sec. III it is explained how the distribution function can be calculated from the conductance by using so-called Ward identities. The general aspects of configurational averaging in the strong scattering regime are discussed in Sec. IV and explicit expressions are derived in the coherent potential approximation in Sec. V. The analytical and numerical calculations are compared in Sec. VI. In Sec. VII the angular dependences of the transmission and reflection probabilities are studied both analytically and numerically. Section VIII presents a discussion of the results including the conductance fluctuations.

\section{TRANSMISSION THROUGH A DISORDERED INTERFACE}

The scattering properties of a single interface for states at the Fermi energy $E_{F}$ can be calculated ${ }^{18}$ directly from the Schrödinger equation

$$
\left[-\frac{\hbar^{2}}{2 m} \nabla^{2}+V(\vec{r})\right] \psi(\vec{r})=E_{F} \psi(\vec{r}) .
$$

The problem can be formulated for arbitrary dimension $d$, so $\vec{r}$ is the $d$-dimensional position vector. The potential on either side of the interface is constant and equal to zero, i.e., a free-electron model is considered and there is no potential step. The scattering potential at the interface is modeled by short-range scatterers with strength $\gamma_{\alpha}$ at position $\vec{\rho}_{\alpha}$ in the plane $x=0$ :

$$
V(\vec{r})=\sum_{\alpha} \gamma_{\alpha} \delta(x) \delta\left(\vec{\rho}-\vec{\rho}_{\alpha}\right)
$$

where $\vec{\rho}$ is the $(d-1)$-dimensional position vector orthogonal to the $x$ direction. The wave function can be expanded in a complete set of transverse plane waves that are labeled by the parallel component of the wave vector $\vec{k}_{\|}$as

$$
\psi(x, \vec{\rho})=\sum_{\vec{k} \|} c_{\vec{k}_{\|}}(x) \frac{e^{i \vec{k} \| \cdot \vec{\rho}}}{\sqrt{A}}
$$

in which $A$ is the $(d-1)$-dimensional cross section of the interface. Employing the orthogonality of the transverse plane waves, a set of one-dimensional equations is obtained

$$
\frac{d^{2}}{d x^{2}} c_{\vec{k}_{\|}}(x)+k_{\perp}^{2} c_{\vec{k}_{\|}}(x)=\frac{2 m}{\hbar^{2}} \sum_{\vec{k}_{\|}^{\prime}} V_{\vec{k}_{\|}, \vec{k}_{\|}^{\prime}} \delta(x) c_{\vec{k}_{\|}^{\prime}}(x),
$$

with

$$
V_{\vec{k}_{\|}, \vec{k}_{\|}^{\prime}}=\sum_{\alpha} \frac{\gamma_{\alpha}}{A} e^{-i\left(\vec{k}_{\|}-\vec{k}_{\|}^{\prime}\right) \cdot \vec{\rho}_{\alpha}}
$$

The perpendicular part of the wave vector $k_{\perp}$ is defined in terms of the Fermi wave vector $k_{F}=\sqrt{2 m E_{F}} / \hbar$ as $k_{\perp}^{2}=k_{F}^{2}$ $-k_{\|}^{2}$. Note that the interface potential gives rise to mixing of different transverse modes. The states with $k_{\|}>k_{F}$ and imaginary $k_{\perp}$ are evanescent (exponentially localized). ${ }^{19}$ The imaginary part of $k_{\perp}$ is chosen to be positive throughout this paper. The solutions for the longitudinal wave functions that are of interest for the transport properties can be written in terms of a propagating incoming state $\vec{k}_{\|}^{\prime}$ and outgoing states $\vec{k}_{\|}$that are either propagating or evanescent:

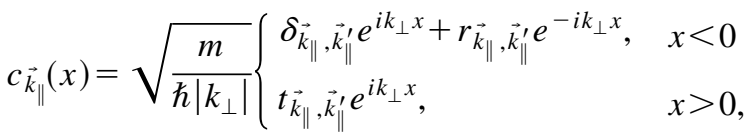

where the matrices $\mathbf{t}$ and $\mathbf{r}$ collect the transmission and reflection amplitudes, respectively. All the propagating states are normalized to carry unit flux perpendicular to the interface. Only the evanescent states that are localized near the interface need to be taken into account (those that are diverging for $x \rightarrow \pm \infty$ have zero amplitude). The transmission and reflection amplitudes can be calculated by matching the wave functions on the two sides of the interface. Continuity of the solution (10) at $x=0$ yields

$$
r_{\vec{k}_{\|}, \vec{k}_{\|}^{\prime}}=t_{\vec{k}_{\|}, \vec{k}_{\|}^{\prime}}-\delta_{\vec{k}_{\|}, \vec{k}_{\|}^{\prime}}
$$

Integration of Eq. (8) over the $\delta$ function gives a relation between the spatial first derivatives of solution (10) on the left- and right-hand sides of the interface. In combination with Eq. (11) this yields an equation for the transmission amplitudes: ${ }^{18}$ 


$$
\sum_{\vec{k}_{\|}^{\prime \prime}}\left[\delta_{\vec{k}_{\|}, \vec{k}_{\|}^{\prime \prime}}+i \Gamma_{\vec{k}_{\|}, \vec{k}_{\|}^{\prime \prime}}\right] t_{\vec{k}_{\|}^{\prime \prime}, \vec{k}_{\|}^{\prime}}=\delta_{\vec{k}_{\|}, \vec{k}_{\|}^{\prime}}
$$

with

$$
\Gamma_{\vec{k}_{\|}, \vec{k}_{\|}^{\prime}}=\frac{m}{\hbar^{2}} \sum_{\alpha} \frac{\gamma_{\alpha}}{A} e^{-i\left(\vec{k}_{\|}-\vec{k}_{\|}^{\prime}\right) \cdot \vec{\rho}_{\alpha}} \frac{\left|k_{\perp}\right|}{k_{\perp}} \frac{1}{\sqrt{\left|k_{\perp}\right|\left|k_{\perp}^{\prime}\right|}} .
$$

This can be written in matrix notation as $[\mathbf{I}+i \boldsymbol{\Gamma}] \mathbf{t}=\mathbf{I}$, with $\mathbf{I}$ the unit matrix. For the transport properties we are only interested in that part of $\mathbf{t}$ that connects propagating states. Separating the outgoing propagating $(p)$ and evanescent $(e)$ states, the matrix equation for $\mathbf{t}$ can be rewritten as

$$
\left(\begin{array}{cc}
\mathbf{I}+i \boldsymbol{\Gamma}_{p p} & i \boldsymbol{\Gamma}_{p e} \\
i \boldsymbol{\Gamma}_{e p} & \mathbf{I}+i \boldsymbol{\Gamma}_{e e}
\end{array}\right)\left(\begin{array}{c}
\mathbf{t}_{p} \\
\mathbf{t}_{e}
\end{array}\right)=\left(\begin{array}{c}
\mathbf{I} \\
\mathbf{0}
\end{array}\right),
$$

with all elements of the matrix $\mathbf{0}$ equal to zero. Elimination of $\mathbf{t}_{e}$ yields $[\mathbf{I}+i \widetilde{\Gamma}] \mathbf{t}_{p}=\mathbf{I}$ with

$$
\widetilde{\Gamma}=\boldsymbol{\Gamma}_{p p}-i \boldsymbol{\Gamma}_{p e}\left[\mathbf{I}+i \boldsymbol{\Gamma}_{e e}\right]^{-1} \boldsymbol{\Gamma}_{e p} .
$$

The Hermitian conjugates of the submatrices of $\boldsymbol{\Gamma}$ are given by $\boldsymbol{\Gamma}_{p p}^{\dagger}=\boldsymbol{\Gamma}_{p p}, \boldsymbol{\Gamma}_{e e}^{\dagger}=-\boldsymbol{\Gamma}_{e e}, \boldsymbol{\Gamma}_{p e}^{\dagger}=i \boldsymbol{\Gamma}_{e p}$ and $\boldsymbol{\Gamma}_{e p}^{\dagger}=i \boldsymbol{\Gamma}_{p e}$, from which it follows that $\widetilde{\Gamma}$ is Hermitian. The transmission amplitudes for the propagating states are thus expressed directly in terms of the scattering potential as

$$
\mathbf{t}_{p}=[\mathbf{I}+i \widetilde{\Gamma}]^{-1}
$$

Using the Hermiticity of $\widetilde{\Gamma}$, the transmission matrix can be written as

$$
\mathbf{t}_{p} \mathbf{t}_{p}^{\dagger}=[\mathbf{I}+\widetilde{\Gamma} \widetilde{\Gamma}]^{-1} .
$$

Equations (16) and (17) are valid for every individual realization of the disorder. Average quantities are obtained by configurational averaging over the random impurity positions that are assumed to be uniformly distributed:

$$
\left\langle a\left(\vec{\rho}_{1}, \ldots, \vec{\rho}_{N_{I}}\right)\right\rangle \equiv \prod_{\alpha=1}^{N_{I}} \int \frac{d \vec{\rho}_{\alpha}}{A} a\left(\vec{\rho}_{1}, \ldots, \vec{\rho}_{N_{I}}\right),
$$

where $N_{I}$ is the number of impurities. This configurational averaging can be carried out both analytically using Greenfunction methods and numerically by brute-force calculations.

\section{WARD IDENTITIES}

The analytical calculation of the configurationally averaged quantities and of the distribution function is based on the expansion of $\mathbf{t}_{p}$ in powers of $\widetilde{\Gamma}$ that follows from Eq. (16):

$$
\mathbf{t}_{p}=\sum_{M=0}^{\infty}(-i \widetilde{\Gamma})^{M}
$$

From this power series one can derive ${ }^{15}$ two so-called Ward identities, which relate single-particle and two-particle properties. The first Ward identity is

$$
\mathbf{t}_{p} \mathbf{t}_{p}^{\dagger}=\frac{1}{2}\left(\mathbf{t}_{p}+\mathbf{t}_{p}^{\dagger}\right)
$$

which follows very generally from current conservation in combination with continuity of the wave function across the interface. A second, less general Ward identity can be derived under the condition that $\widetilde{\Gamma}$ is proportional to $m$ :

$$
\mathbf{t}_{p} \mathbf{t}_{p}=\left(1+m \frac{\partial}{\partial m}\right) \mathbf{t}_{p}
$$

This relation is valid when evanescent states can be disregarded, which is the case in the limit of weak scattering. However, also in the strong scattering regime $\widetilde{\Gamma}$ is proportional to $m$, as can be seen from Eqs. (13) and (15). The Ward identity (21) thus applies both in the weak scattering regime (considered in Ref. 15) and in the strong scattering regime (considered in Ref. 17), but not in the intermediate regime. Note that Eq. (21) can also be expressed in terms of the derivative with respect to the (average) scattering strength. ${ }^{15}$ Although both alternatives should be equivalent, the expression in terms of the mass leads to more transparent results in the dirty limit.

Using these two Ward identities and their Hermitian conjugates repeatedly, higher-order products of $\mathbf{t}_{p}$ and $\mathbf{t}_{p}^{\dagger}$ can be reduced to expressions that contain only $\mathbf{t}_{p}$ and $\mathbf{t}_{p}^{\dagger}$. This simplifies the calculations enormously. The distribution function $P(T)$ can be rewritten in terms of a power series in the transmission matrix by expressing the $\delta$ function in Eq. (1) as a Fourier integral and subsequently expanding the exponent $\exp \left(i q \mathbf{t}_{p} \mathbf{t}_{p}^{\dagger}\right)$ :

$$
P(T)=\int \frac{d q}{2 \pi} e^{-i q T} \sum_{n=0}^{\infty} \frac{(i q)^{n}}{n !} \operatorname{Tr}\left\langle\left(\mathbf{t}_{p} \mathbf{t}_{p}^{\dagger}\right)^{n}\right\rangle .
$$

By using the Ward identities repeatedly and applying a Kramers-Kronig relation in the parameter $\eta=m^{2}$, this expression can for an integrable function $\langle g(\eta)\rangle=\operatorname{Tr}\left\langle\mathbf{t}_{p} \mathbf{t}_{p}^{\dagger}\right\rangle$ be rewritten as ${ }^{15}$

$$
P(T)=\frac{1}{\pi} \frac{1}{T(1-T)} \operatorname{Im}\left[\left\langle g\left(\frac{\eta T}{T-1}-i 0^{+}\right)\right\rangle,\right.
$$

with $0^{+}$a positive infinitesimal. Details of the derivation of Eq. (23) and a discussion of a possibly nonintegrable part of $\langle g(\eta)\rangle$ are given in the Appendix. By calculating $\left\langle\mathbf{t}_{p}\right\rangle$ we obtain the conductance from

$$
\langle g(\eta)\rangle=\operatorname{Re}\left[\operatorname{Tr}\left\langle\mathbf{t}_{p}\right\rangle\right]
$$

The calculation of the distribution function is thus reduced to the calculation of the configurational average of the transmission amplitude matrix.

\section{CONFIGURATIONAL AVERAGING}

In order to calculate the configurational average of $\mathbf{t}_{p}$ it is convenient to introduce the Green-function matrix $\mathbf{G}^{+}$. The elements of $\mathbf{G}^{+}$are related to the transmission amplitudes by $^{20}$ 
(a)

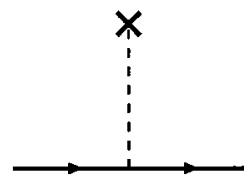

(b)

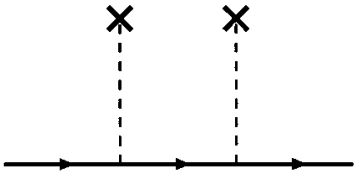

(c)

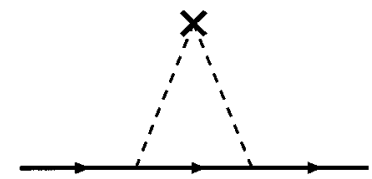

(d)
FIG. 1. Lowest-order diagrams in the expansion for the configurationally averaged Green function. The solid lines with arrows represent unperturbed Green functions, the crosses are the scattering centers, and each dashed line is a scattering event. (a) and (b) are the zeroth- and first-order diagrams, respectively. The secondorder diagrams (c) and (d) represent scattering once at two different impurities and scattering twice at a single impurity, respectively.

$$
t_{\vec{k}_{\|}, \vec{k}_{\|}^{\prime}}=i \frac{\hbar^{2}}{m} \sqrt{k_{\perp} k_{\perp}^{\prime}} G_{\vec{k}_{\|}, \vec{k}_{\|}^{\prime}}^{+}
$$

The unperturbed Green-function matrix $\mathbf{G}^{+(0)}$ is diagonal and its elements are given by

$$
G_{\vec{k}_{\|}, \vec{k}_{\|}^{\prime}}^{+(0)}=G_{\vec{k}_{\|}}^{+(0)} \delta_{\vec{k}_{\|}, \vec{k}_{\|}^{\prime}}=-i \frac{m}{\hbar^{2}} \frac{1}{k_{\perp}} \delta_{\vec{k}_{\|}, \vec{k}_{\|}^{\prime}}
$$

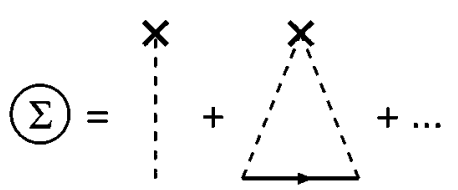

FIG. 2. Lowest-order irreducible diagrams that contribute to the self-energy, which is represented by the big circle with the $\Sigma$ inside.

From Eqs. (19) and (25) an expansion of the configurationally averaged Green function in terms of the scattering potential is obtained, which reads

$$
\left\langle G_{\vec{k}_{\|}, \vec{k}_{\|}}^{+}\right\rangle=-i \frac{m}{\hbar^{2}} \frac{1}{\sqrt{k_{\perp} k_{\perp}^{\prime}}} \sum_{N=0}^{\infty}\left\langle(-i \widetilde{\Gamma})^{N}\right\rangle_{\vec{k}_{\|}, \vec{k}_{\|}^{\prime}}
$$

The different terms in this expansion are obtained by integration over all possible impurity positions and can be represented by Feynman diagrams. ${ }^{18}$ The zeroth-order term is given by

$$
-i \frac{m}{\hbar^{2}} \frac{1}{\sqrt{k_{\perp} k_{\perp}^{\prime}}}(-i)^{0}\left\langle\widetilde{\Gamma}^{0}\right\rangle_{\vec{k}_{\|}, \vec{k}_{\|}^{\prime}}=G_{\vec{k}_{\|}}^{+(0)} \delta_{\vec{k}_{\|}, \vec{k}_{\|}^{\prime}}
$$

which is nothing but the unperturbed Green function, depicted schematically in Fig. 1(a). To first order in the scattering potential only the propagating states play a role:

$$
-i \frac{m}{\hbar^{2}} \frac{1}{\sqrt{k_{\perp} k_{\perp}^{\prime}}}(-i)\left\langle\boldsymbol{\Gamma}_{p p}\right\rangle_{\vec{k}_{\|}, \vec{k}_{\|}^{\prime}}=G_{\vec{k}_{\|}}^{+(0)}\left(\sum_{\alpha} \frac{\gamma_{\alpha}}{A}\right) G_{\vec{k}_{\|}}^{+(0)} \delta_{\vec{k}_{\|}, \vec{k}_{\|}^{\prime}} \cdot
$$

This term comes from scattering once at a single impurity and is represented by the diagram depicted in Fig. 1(b). In the second-order term also the evanescent states should be taken into account:

$$
\begin{gathered}
-i \frac{m}{\hbar^{2}} \frac{1}{\sqrt{k_{\perp} k_{\perp}^{\prime}}}(-i)^{2}\left\langle\boldsymbol{\Gamma}_{p p}^{2}+\boldsymbol{\Gamma}_{p e} \boldsymbol{\Gamma}_{e p}\right\rangle_{\vec{k}_{\|}, \vec{k}_{\|}^{\prime}}=G_{\vec{k}_{\|}}^{+(0)}\left(\sum_{\alpha} \frac{\gamma_{\alpha}}{A}\right) G_{\vec{k}_{\|}}^{+(0)}\left(\sum_{\alpha^{\prime}} \frac{\gamma_{\alpha^{\prime}}}{A}\right) G_{\vec{k}_{\|}}^{+(0)} \delta_{\vec{k}_{\|}, \vec{k}_{\|}^{\prime}} \\
+G_{\vec{k}_{\|}}^{+(0)}\left[\sum_{\alpha}\left(\frac{\gamma_{\alpha}}{A}\right)^{2}\right]\left(\sum_{\vec{k}_{\|}^{\prime \prime}} G_{\vec{k}_{\|}^{\prime \prime}}^{+(0)}\right) G_{\vec{k}_{\|}}^{+(0)} \delta_{\vec{k}_{\|}, \vec{k}_{\|}^{\prime}}
\end{gathered}
$$

The first term stems from scattering once at two different scatterers, whereas the second term arises from scattering twice at the same scatterer. The corresponding diagrams are shown in Figs. 1(c) and 1(d). Note that the evanescent states enter only via the intermediate wave-vector summation. The higher order terms can be represented by Feynman diagrams according to the following set of rules. ${ }^{18}$

(i) For each electron line, introduce $G_{\vec{k}_{\|}}^{+(0)}$.

(ii) For each scattering vertex $\alpha$, introduce $\gamma_{\alpha} / A$.

(iii) Conserve momentum at each vertex.

(iv) Sum over all impurities. (v) Sum over all intermediate states, including evanescent states.

The exact configurationally averaged Green function can be obtained by summing over all diagrams. These can be partially summed by introducing the irreducible self-energy. An irreducible diagram is a diagram that cannot be divided into two subdiagrams joined only by a single electron line. Examples of irreducible diagrams are shown in Fig. 2. Because the translation invariance parallel to the interface is restored after configurational averaging, the self-energy matrix $\Sigma$ is diagonal in $\vec{k}_{\|}$: 


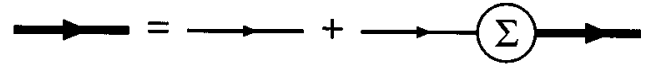

FIG. 3. Schematic representation of the Dyson equation, which relates the perturbed Green function (thick line) to the unperturbed Green function (thin line) and the self-energy (circle).

$$
\Sigma_{\vec{k}_{\|}, \vec{k}_{\|}^{\prime}}=\Sigma_{\vec{k}_{\|}} \delta_{\vec{k}_{\|}}, \vec{k}_{\|}^{\prime}
$$

The Dyson equation $\left\langle\mathbf{G}^{+}\right\rangle=\mathbf{G}^{+(0)}+\mathbf{G}^{+(0)} \mathbf{\Sigma}\left\langle\mathbf{G}^{+}\right\rangle$relates the configurationally averaged Green function to the unperturbed Green function and the self-energy. A schematic representation of this equation is given Fig. 3. One can easily see that substitution of the self-energy of Fig. 2 into the Dyson equation of Fig. 3 generates the reducible diagrams of Fig. 1. Substitution of Eqs. (26) and (31) into the Dyson equation yields

$$
\left\langle G_{\vec{k}_{\|}, \vec{k}_{\|}}^{+}\right\rangle=\left\langle G_{\vec{k}_{\|}}^{+}\right\rangle \delta_{\vec{k}_{\|}, \vec{k}_{\|}^{\prime}}=\frac{1}{\left(G_{\vec{k}_{\|}}^{+(0)}\right)^{-1}-\Sigma_{\vec{k}_{\|}}} \delta_{\vec{k}_{\|}, \vec{k}_{\|}^{\prime}} \cdot
$$

The Green function is thus diagonal in $\vec{k}_{\|}$after configurational averaging. In the strong scattering regime where $g$ $\ll N$ the self-energy is much larger than the inverse of the unperturbed Green function and Eq. (32) can be expanded as

$$
\left\langle G_{\vec{k}_{\|}}^{+}\right\rangle=-\frac{1}{\sum_{\vec{k}_{\|}}}\left[1+\frac{1}{G_{\vec{k}_{\|}}^{+(0)} \Sigma_{\vec{k}_{\|}}}+\cdots\right] .
$$

The leading term in the expansion of the Green function thus only depends on the self-energy; it does not contain the unperturbed Green function and therefore does not depend on the effective mass.

In the complete perturbation expansion of the irreducible self-energy all internal Green functions are fully renormalized, i.e., in all the intermediate wave-vector summations the unperturbed Green function is replaced by the perturbed Green function. In the strong scattering regime the perturbed Green function depends only on the self-energy. It then follows directly from the Feynman rules that the summation over any set of renormalized diagrams leads to a selfconsistent equation for the self-energy that does not contain the unperturbed Green function and is thus independent of the electron mass $m$. The simple and (in the present model) exact result for the distribution function in the strong scattering regime as derived below is a direct consequence of this $m$ independence of the self-energy.

In the strong scattering regime the average conductance can be calculated from the self-energy using Eqs. (24), (25), and (33) as

$$
\langle g(\eta)\rangle=\frac{\hbar^{2}}{\sqrt{\eta}} \sum_{\vec{k}_{\|}}^{(p)} \frac{-\operatorname{Im}\left[\Sigma_{\vec{k}_{\|}}\right] k_{\perp}}{\left|\Sigma_{\vec{k}_{\|}}\right|^{2}}
$$

and is thus proportional to $1 / \sqrt{\eta}=1 / \mathrm{m}$. The summation over $\vec{k}_{\|}$is restricted to the propagating modes $(p)$. Substitution of Eq. (34) into Eq. (23) and using $g(\infty)=0$ yields

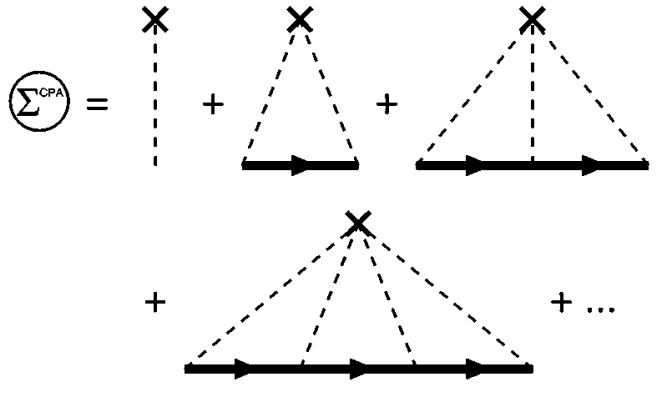

FIG. 4. Irreducible diagrams that are taken into account in the calculation of the self-energy in the coherent potential approximation.

$$
P(T)=\frac{\langle g\rangle}{\pi} \frac{1}{T^{3 / 2} \sqrt{1-T}} \quad \text { for } \frac{1}{1+\left(\frac{\pi N}{2\langle g\rangle}\right)^{2}}<T<1
$$

and $P(T)=0$ otherwise. The cutoff at small $T$ is introduced to ensure that $\int_{0}^{1} d T P(T)=N$; the origin of this cutoff is discussed in the Appendix. For $N \gg g$ this normalization is irrelevant for the averages of the first- and higher-order moments of $T$. We emphasize that Eq. (35) is valid only in the strong scattering regime where $g \ll N$.

The distribution function of transmission matrix eigenvalues for a strongly disordered interface is thus universal in the sense that it does not depend on any of the microscopic parameters or on the dimension $d$; it depends only on the macroscopic conductance. We obtained this result without explicitly calculating any Feynman diagrams, only using the general property that the self-energy is independent of the electron mass. Note that Eq. (35) differs significantly from the universal result (4) obtained for diffusive bulk conductors in the weak scattering regime.

Also for dirty interfaces universality has its limits. When the self-energy has no imaginary part the conductance vanishes in lowest order and the calculation yields $P(T)$ $=N \delta(T)$. A real self-energy is, for example, characteristic for a tunnel barrier. To obtain a finite conductance in that case higher-order terms in the expansion (33) should be taken into account, which give rise to nonuniversal results. This loss of universality is analogous to the situation considered by Nazarov, ${ }^{13}$ who finds that universal behavior in bulk disordered conductors can be destroyed by a tunnel barrier.

\section{COHERENT POTENTIAL APPROXIMATION}

To be more specific we will now explicitly calculate the self-energy for a limited set of diagrams, namely, all diagrams in which the lines representing potential scattering do not cross. This corresponds to calculating the scattering at a single impurity exactly while treating the scattering from all the other impurities in a mean-field approximation. Quantum interference of electron waves scattered from different impurities is thus disregarded. The diagrams that are taken into account are shown in Fig. 4. Using the Dyson equation of Fig. 3 one can easily verify that all noncrossed diagrams are included in this way. This approximation is known as the single-site coherent potential approximation (CPA). The self- 


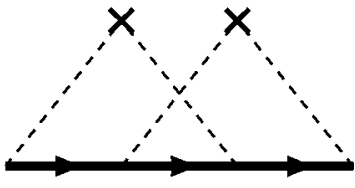

FIG. 5. Example of a crossed diagram. Crossed diagrams are not taken into account in the coherent potential approximation.

energy is obtained by summing over all diagrams of Fig. 4:

$$
\Sigma^{\mathrm{CPA}}=\sum_{\alpha} \frac{\gamma_{\alpha}}{A} \sum_{M=0}^{\infty}\left(\frac{\gamma_{\alpha}}{A} \sum_{\vec{k} \|}\left\langle G_{\vec{k}_{\|}}^{+}\right\rangle\right)^{M},
$$

which does not depend on $\vec{k}_{\|}$. Substitution of the lowestorder term of Eq. (33) yields a self-consistent equation for the self-energy. For $\gamma_{\alpha}= \pm \gamma$ the self-energy in the strong scattering limit is given by

$$
\Sigma_{\infty}^{\mathrm{CPA}}=\frac{1}{2 A}\left[N_{I} \bar{\gamma}-i \sqrt{4\left(N_{I}-N_{T}\right) N_{T} \gamma^{2}-N_{I}^{2} \bar{\gamma}^{2}}\right],
$$

where $N_{T}$ is the total number of propagating plus evanescent states. The average of the scattering strengths is $\bar{\gamma}$ $=\Sigma_{\alpha} \gamma_{\alpha} / N_{I}$. Note that $\Sigma_{\infty}^{\mathrm{CPA}}$ does indeed not depend on $m$. In order to obtain nontrivial results the imaginary part of $\Sigma_{\infty}^{\mathrm{CPA}}$ should not vanish. In the strong scattering regime this is the case when the number of scatterers is larger than the total number of channels, i.e., $N_{I}>N_{T}$. We therefore can allow only a finite number of evanescent states. It is indeed well known in scattering theory that the cross section of $\delta$ scatterers vanishes without such a cutoff. Another requirement for a nonvanishing imaginary part of $\Sigma_{\infty}^{\mathrm{CPA}}$ is a limited average scattering strength, i.e., $(\bar{\gamma} / \gamma)^{2}$ $<4\left(N_{I}-N_{T}\right) N_{T} / N_{I}^{2}$. When $\operatorname{Im}\left[\sum_{\infty}^{\mathrm{CPA}}\right] \neq 0$ the average conductance can be calculated from Eq. (34):

$$
\langle g\rangle=\frac{A \sum_{\vec{k} \|}^{(p)} k_{\perp}}{\pi \tilde{\gamma} \sqrt{\left(N_{I}-N_{T}\right) N_{T}}} \sqrt{1-\frac{\bar{\gamma}^{2}}{\gamma^{2}} \frac{N_{I}^{2}}{4\left(N_{I}-N_{T}\right) N_{T}}},
$$

where we introduced the normalized scattering strength $\tilde{\gamma}$ $=m \gamma / \hbar^{2} \pi$, which is a dimensionless quantity for $d=2$. The summation of $k_{\perp}$ over the propagating modes depends on the dimension: It equals $\pi / 4 N k_{F}$ for $d=2$ and $\frac{2}{3} N k_{F}$ for $d=3$.

Crossed diagrams are not included in the CPA, but they can be important in the strong scattering regime. The contribution to the self-energy of, for example, the crossed diagram depicted in Fig. 5 equals

$$
\sum_{\alpha, \alpha^{\prime}}\left(\frac{\gamma_{\alpha}}{A} \frac{\gamma_{\alpha^{\prime}}}{A}\right)^{2} \sum_{\vec{k}_{\|}^{\prime}, \vec{k}_{\|}^{\prime \prime}}\left\langle G_{\vec{k}_{\|}^{\prime}}^{+}\right\rangle\left\langle G_{\vec{k}_{\|}^{\prime \prime}}^{+}\right\rangle\left\langle G_{\vec{k}_{\|}-\vec{k}_{\|}^{\prime}+\vec{k}_{\|}^{\prime \prime}}^{+}\right\rangle .
$$

The summation over the intermediate states in Eq. (39) is restricted by the cutoff in the integral over evanescent states in combination with the momentum conservation at each vertex. Because of this restriction the self-energy depends on $\vec{k}_{\|}$. A simple estimate shows that the contribution to the self-energy of the crossed diagram of Fig. 5 is of the same order of magnitude as the contribution of the second noncrossed diagram in Fig. 4. There is thus no a priori justification for the omission of the crossed diagrams in the strong scattering regime. Substantial errors in the CPA caused by the neglect of quantum interference therefore cannot be excluded.

\section{NUMERICAL RESULTS}

In order to confirm the analytical calculations in the dirty limit and explore the not-so-dirty regime, we perform the ensemble averaging numerically by brute-force calculations of many realizations of the impurity positions. These calculations are limited to $d=2$ (a two-dimensional "impurity necklace, $\left.{ }^{\prime \prime}\right)$ and $\gamma_{\alpha}= \pm \gamma$. To compare with the analytical results in the strong scattering regime the conductance should be sufficiently smaller than the number of conduction channels. On the other hand, the conductance should not be too small to avoid that the number of eigenvalues close to 1 becomes so low that large statistical fluctuations occur. Most of our calculations are for $0.005<g / N<0.030$. Whereas the analytical calculations are in the limit $N \rightarrow \infty$, the numerical calculations are for a finite number of conduction channels that may give rise to finite-size corrections. The results presented in this section are obtained for $N=20$ channels, which are consistent with those for $N$ up to 80 (see Sec. VIII). All configurational averages were calculated using an ensemble of 10000 independent realizations of the impurity positions.

The eigenvalues of the transmission matrix can be obtained by calculating the eigenvalues of the matrix $\mathbf{I}+\widetilde{\Gamma} \widetilde{\Gamma}$ that are equal to $1 / T_{n}$, as can be seen from Eq. (17). The distribution function is not a convenient function to compute because of the divergences at $T=0$ and $T=1$. Instead of $P(T)$ we calculate the well-behaved integrated quantity

$$
Q(T)=\frac{1}{\langle g\rangle} \int_{0}^{T} d T^{\prime} T^{\prime} P\left(T^{\prime}\right),
$$

which is a smooth function of $T$. $Q(T)$ is the relative contribution to the conductance of all $T_{n}<T$. From Eqs. (4) and (35) it follows that $Q(T)=1-\sqrt{1-T}$ for a disordered bulk conductor and $Q(T)=(1 / \pi) \arccos (1-2 T)$ for a dirty interface. In Fig. 6 numerically calculated $Q(T)$ for three different sets of microscopic parameters are compared with the analytical results. Figure 6 shows excellent agreement between analytical and numerical configurational averages. Note that the numerical results differ significantly from the analytical result for disordered bulk systems.

In order to appreciate the limits of the universality and test the CPA we study numerically the dependence of the conductance and the distribution function on the different microscopic parameters. Instead of calculating the functions $P(T)$ or $Q(T)$ we compute only the average values of three physical quantities that depend characteristically on $P(T)$. In Table I the expectation values for the conductance $g$ and the shot-noise power $p$ as well as for the conductance $g_{\text {NS }}$ and the shot-noise power $p_{\mathrm{NS}}$ of a normal metal/superconductor junction are given for both a dirty interface and a disordered bulk conductor. These expectation values are sufficient to discriminate between the two universal distributions. Figure 7 shows the dependence of the average conductance and the 


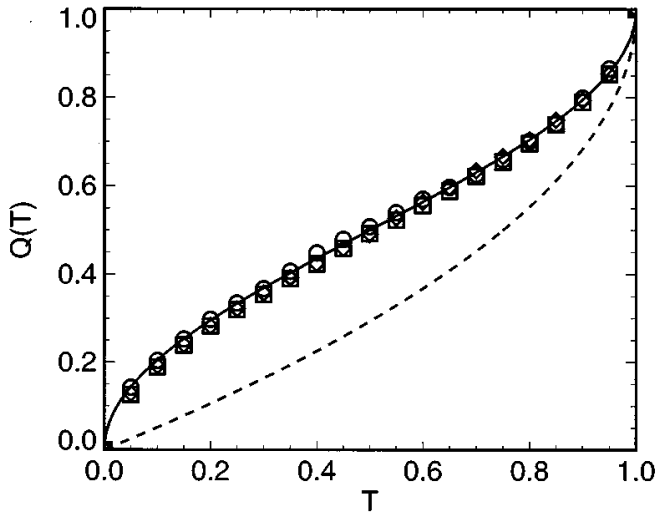

FIG. 6. Function $Q(T)$ obtained from three different numerical calculations (symbols) and from the analytical results for a dirty interface (solid line) and for a disordered bulk conductor (dashed line). The numerical calculation were carried out using $N=20, N_{I}$ $=200, \bar{\gamma}=0$, and $N_{T}=20, \tilde{\gamma}=10$ for the squares, $N_{T}=20, \tilde{\gamma}=60$ for the circles, and $N_{T}=40, \tilde{\gamma}=10$ for the diamonds. Configurational averaging was done using 10000 realizations of the disorder.

ratios $\langle p\rangle /\langle g\rangle,\left\langle g_{\mathrm{NS}}\right\rangle /\langle g\rangle$, and $\left\langle p_{\mathrm{NS}}\right\rangle /\langle g\rangle$ on the dimensionless scattering strength $\tilde{\gamma}$. Even though the conductance changes as a function of $\tilde{\gamma}$, the ratios characterizing the distribution function remain constant which confirms the universality of $P(T)$. The numerically calculated average conductance deviates from the result obtained in the CPA. This is not unexpected since in the CPA the crossed diagrams are not taken into account. The difference is, however, surprisingly small. A good fit to the numerical calculations can be obtained by simply multiplying the CPA results by a scaling factor of order 1. In the present regime the crossed diagrams thus only weakly renormalize the CPA result.

In Figs. 8 and 9 the average conductance and the ratios characterizing the distribution function are plotted as functions of the number of scatterers $N_{I}$ and the total number of modes $N_{T}$, respectively. The results confirm that $P(T)$ is universal and that $\langle g\rangle$ follows the behavior obtained from the CPA up to a scaling factor. Note that the same scaling factor is used in Figs. 7-9.

Finally, we consider the dependences on the average scattering strength $\bar{\gamma}$. Figure 10 shows that the average conductance as a function of $\bar{\gamma} / \gamma$ differs qualitatively from the CPA

TABLE I. Expectation values of the properties $a$ that are described by the linear statistics $a(T)$ for the distribution function of a strongly disordered interface and of a metallic bulk system. The physical properties considered are the conductance $g$ and the shotnoise power $p$ as well as the conductance $g_{\mathrm{NS}}$ and the shot-noise power $p_{\mathrm{NS}}$ of a normal metal/superconductor junction.

\begin{tabular}{lccc}
\hline \hline$a$ & & $\langle a\rangle /\langle g\rangle$ & $\langle a\rangle /\langle g\rangle$ \\
& $a(T)$ & interface & bulk \\
\hline$g$ & $T$ & 1 & 1 \\
$p$ & $T(1-T)$ & $\frac{1}{2}$ & $\frac{1}{3}$ \\
$g_{\text {NS }}$ & $2 T^{2} /(2-T)^{2}$ & $\frac{1}{2} \sqrt{2}$ & 1 \\
$p_{\text {NS }}$ & $16 T^{2}(1-T) /(2-T)^{4}$ & $\frac{3}{8} \sqrt{2}$ & $\frac{2}{3}$ \\
\hline \hline
\end{tabular}

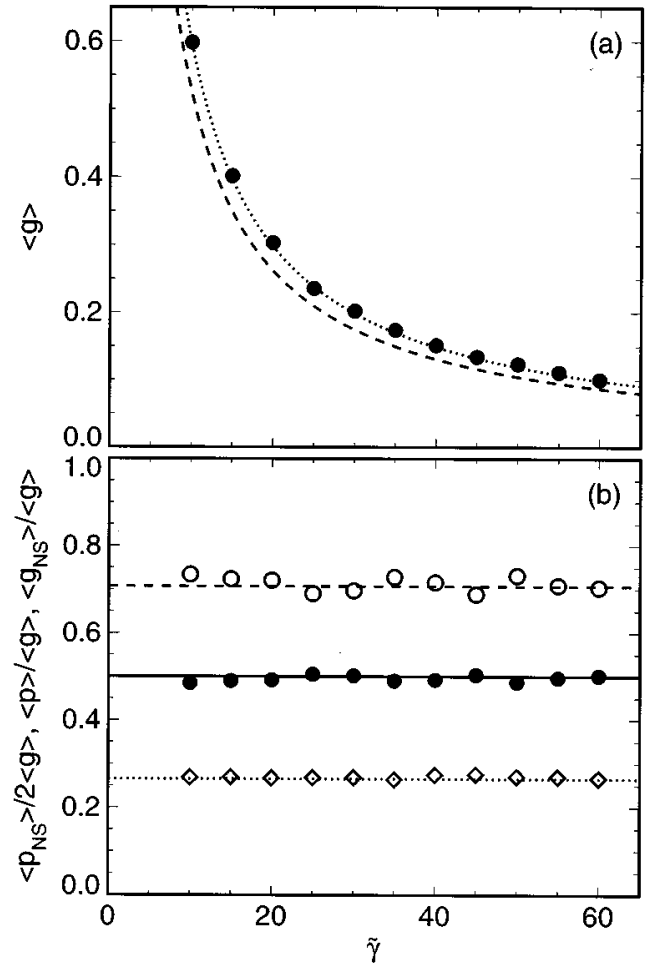

FIG. 7. Dependence of (a) the average conductance $\langle g\rangle$ and (b) the ratios $\langle p\rangle /\langle g\rangle,\left\langle g_{\mathrm{NS}}\right\rangle /\langle g\rangle$ and $\left\langle p_{\mathrm{NS}}\right\rangle / 2\langle g\rangle$ on the dimensionless scattering strength $\widetilde{\gamma}$. The numerical calculations were carried out using $N=20, N_{T}=20, N_{I}=200$, and $\bar{\gamma}=0.0$. Configurational averaging was done using 10000 realizations of the disorder. (a) The numerically calculated values of $\langle g\rangle$ (filled circles) compared with the CPA result (dashed line) and with the CPA result multiplied by a factor 1.14 (dotted line). (b) The numerically calculated values of the ratios $\langle p\rangle /\langle g\rangle$ (filled circles), $\left\langle g_{\mathrm{NS}}\right\rangle /\langle g\rangle$ (open circles), and $\left\langle p_{\mathrm{NS}}\right\rangle / 2\langle g\rangle$ (diamonds) compared with their universal values given by the solid, dashed, and dotted lines, respectively.

calculation, in contrast to the results for $\bar{\gamma}=0$ where they differed only by a scaling factor close to unity. More importantly, the universality breaks down. Whereas the distribution function remains universal for $\bar{\gamma} / \gamma<0.6$, it becomes nonuniversal for $\bar{\gamma} / \gamma>0.6$. The value at which the ratios characterizing $P(T)$ start to deviate from the universal values coincides with the value at which the CPA conductance vanishes. Nonuniversal behavior must be caused by higherorder terms in Eq. (33). The numerical results thus indicate that for $\bar{\gamma} / \gamma>0.6$ the crossed diagrams do not contribute to the imaginary part of the self-energy to lowest order in the expansion of the Green function. A more detailed study of breakdown of universality is beyond the scope of the present paper, but we note that a similar effect has been found for disordered bulk systems in the presence of a tunnel barrier. ${ }^{13}$ For $\bar{\gamma} / \gamma=1$ the numerical results in Fig. 10 indeed become characteristic for a tunnel barrier.

In both the analytical and the numerical calculations presented above the disorder is due to the random positions of the scatterers. We carried out additional numerical calculations in which also the scattering strengths $\gamma_{\alpha}$ were chosen at random, in contrast to the constant value for $\left|\gamma_{\alpha}\right|$ used in the 


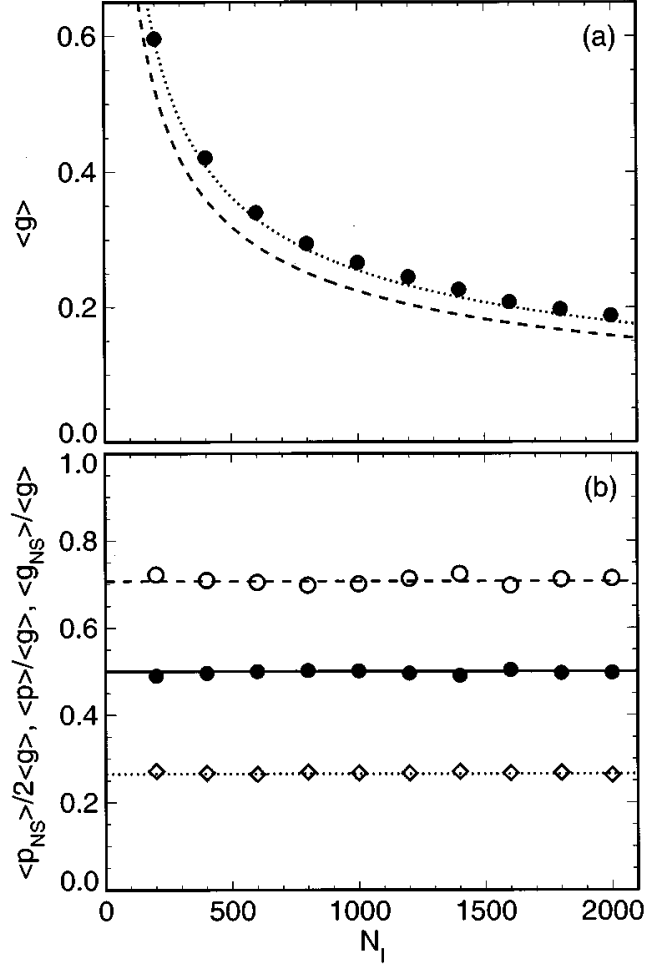

FIG. 8. Dependence of (a) the average conductance $\langle g\rangle$ and (b) the ratios $\langle p\rangle /\langle g\rangle,\left\langle g_{\mathrm{NS}}\right\rangle /\langle g\rangle$, and $\left\langle p_{\mathrm{NS}}\right\rangle / 2\langle g\rangle$ on the number of scatterers $N_{I}$. The numerical calculations were carried out using $N=20, N_{T}=20, \tilde{\gamma}=10.0$, and $\bar{\gamma}=0.0$. Configurational averaging was done using 10000 realizations of the disorder. For explanation of the symbols and the lines, see Fig. 7.

other calculations of this section. Also with random scattering strengths the universal distribution function (35) persists, as expected.

Whereas the analytical results of this paper are restricted to the strong scattering regime with $g \ll N$, the numerical calculations can straightforwardly be carried out for arbitrary values of $g$. In Fig. 11 the conductance and the ratios characterizing the distribution function are plotted as a function of the number of scatterers $N_{I}$. For $N_{I} \gg N$ the results are in the strong scattering regime. For $N_{I} \ll N$ the weak scattering regime is reached where $g \leq N$. For the value $\tilde{\gamma}=10.0$ used in the calculations presented in Fig. 11, the regime $N_{I} \ll N$ corresponds the limit of a few strong scatterers that was considered in Ref. 15. In this limit analytical calculations ${ }^{15}$ yield $N_{I}$ eigenstates that are completely reflected $\left(T_{n}=0\right)$, whereas the remaining $N-N_{I}$ states are completely transmitted $\left(T_{n}=1\right)$, which is consistent with the numerical results for $N_{I} \ll N$ in Fig. 11. In between the weak and strong scattering regimes a smooth transition is observed.

\section{TRANSMISSION PROBABILITIES}

Next we calculate the configurationally averaged transmission probabilities

$$
\left\langle\left|t_{\vec{k}_{\|}, \vec{k}_{\|}^{\prime}}\right|^{2}\right\rangle=\frac{\hbar^{4}}{m^{2}} k_{\perp} k_{\perp}^{\prime}\left\langle G_{\vec{k}_{\|}, \vec{k}_{\|}^{\prime}}^{+} G_{\vec{k}_{\|}, \vec{k}_{\|}^{\prime}}^{+*}\right\rangle
$$

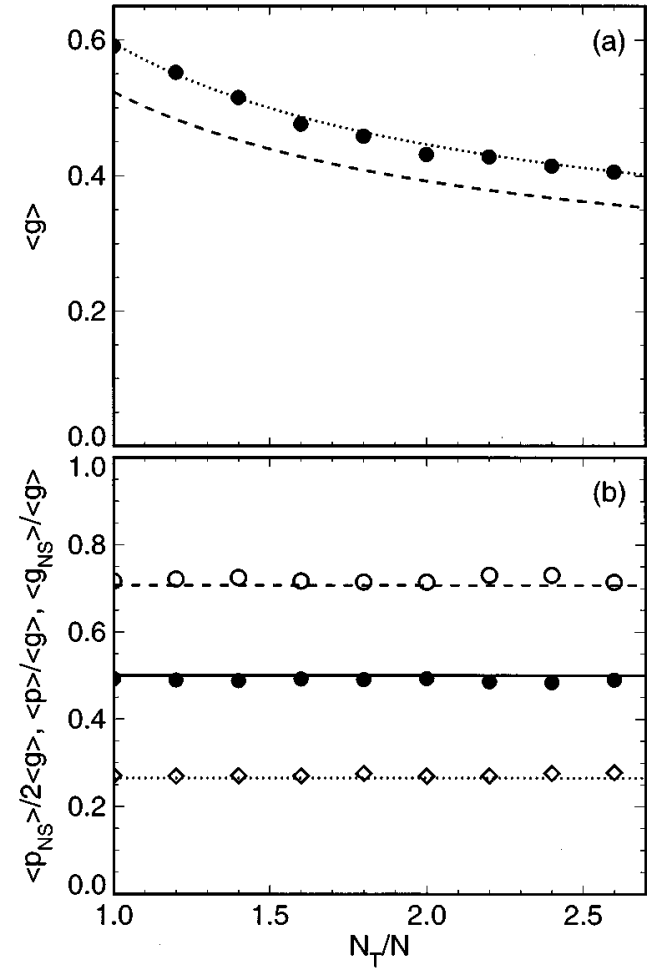

FIG. 9. Dependence of (a) the average conductance $\langle g\rangle$ and (b) the ratios $\langle p\rangle /\langle g\rangle,\left\langle g_{\mathrm{NS}}\right\rangle /\langle g\rangle$, and $\left\langle p_{\mathrm{NS}}\right\rangle / 2\langle g\rangle$ on the total number of propagating and evanescent states $N_{T}$. The numerical calculations were carried out using $N=20, N_{I}=200, \tilde{\gamma}=10.0$ and $\bar{\gamma}$ $=0.0$. Configurational averaging was done using 10000 realizations of the disorder. For explanation of the symbols and the lines, see Fig. 7.

The product of the electron and the hole Green function in Eq. (41) cannot be reduced to a one-particle Green function by the Ward identities, as was the case in the calculation of the average conductance $\langle g\rangle$. Instead, the expectation value of the product has to be calculated explicitly. The configurationally averaged two-particle Green function can be written as

$$
\left\langle\left|G_{\vec{k}_{\|}, \vec{k}_{\|}}^{+}\right|^{2}\right\rangle=\left|\left\langle G_{\vec{k}_{\|}}^{+}\right\rangle\right|^{2} \delta_{\vec{k}_{\|}, \vec{k}_{\|}^{\prime}}+\left|\left\langle G_{\vec{k}_{\|}}^{+}\right\rangle\right|^{2} W_{\vec{k}_{\|}, \vec{k}_{\|}^{\prime}}\left|\left\langle G_{\vec{k}_{\|}^{\prime}}^{+}\right\rangle\right|^{2},
$$

with $\mathbf{W}$ the reducible vertex function matrix. $\mathbf{W}$ contains all the correlated two-particle diagrams in which the electron and the hole line are connected by a scattering vertex.

To keep the analytical calculations tractable, we will restrict ourselves to the self-consistent Born approximation (SCBA). In the SCBA calculation of the self-energy only the first two diagrams of Fig. 4 are taken into account, which in the strong scattering limit yields

$$
\sum_{\infty}^{\mathrm{SCBA}}=\frac{1}{2 A}\left[N_{I} \bar{\gamma}-i \sqrt{4 N_{I} N_{T} \gamma^{2}-N_{I}^{2} \bar{\gamma}^{2}}\right]
$$

The restriction $\gamma_{\alpha}= \pm \gamma$ that was used in the CPA can be eliminated and $\gamma^{2}$ now equals the mean-square value of the scattering strengths: $\gamma^{2}=\Sigma_{\alpha} \gamma_{\alpha}^{2} / N_{I}$. Note that in the limit $N_{I} \gg N_{T}$ for $\gamma_{\alpha}= \pm \gamma$ the higher-order diagrams in Fig. 4 can be neglected and the CPA reduces to the SCBA. Crossed 


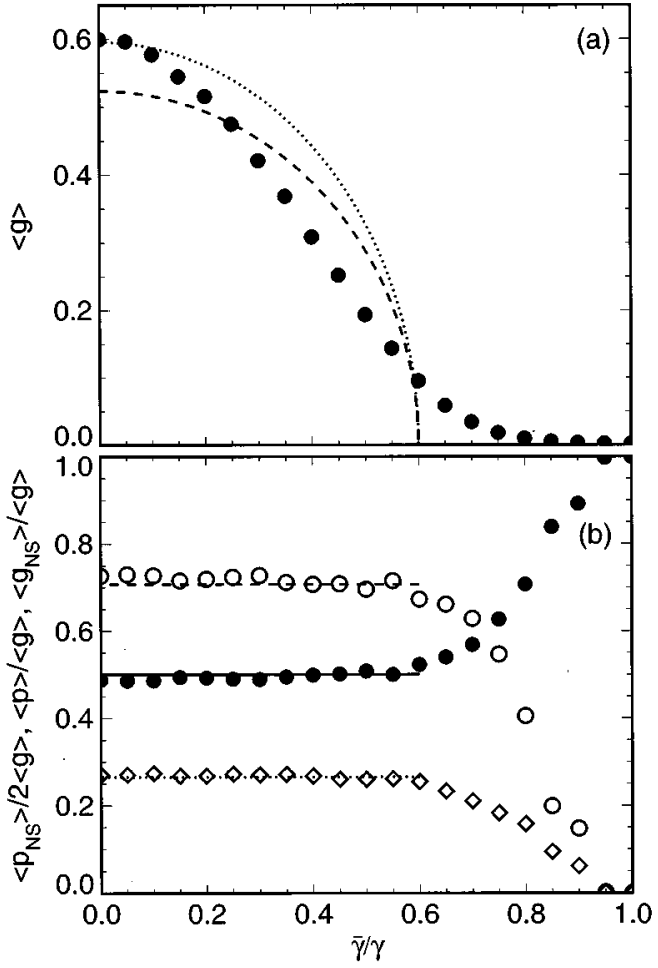

FIG. 10. Dependence of (a) the average conductance $\langle g\rangle$ and (b) the ratios $\langle p\rangle /\langle g\rangle,\left\langle g_{\mathrm{NS}}\right\rangle /\langle g\rangle$, and $\left\langle p_{\mathrm{NS}}\right\rangle / 2\langle g\rangle$ on the relative average scattering strength $\bar{\gamma} / \gamma$. The numerical calculations were carried out using $N=20, N_{T}=20, N_{I}=200$, and $\tilde{\gamma}=10.0$. Configurational averaging was done using 10000 realizations of the disorder. For explanation of the symbols and the lines, see Fig. 7.

diagrams such as the one in Fig. 5 are neglected, however, in spite of being of the same order as those taken into account in the SCBA.

The diagrams for the reducible vertex function in the SCBA are shown in Fig. 12. These ladder diagrams are independent of the incoming and outgoing modes and can be easily summed:

$$
W_{\vec{k}_{\|}, \vec{k}_{\|}^{\prime}}=W=\frac{\sigma}{1-\sigma \sum_{\vec{k}_{\|}^{\prime \prime}}\left|\left\langle G_{\vec{k}_{\|}^{\prime \prime}}^{+}\right\rangle\right|^{2}},
$$

where $\sigma=N_{I} \gamma^{2} / A^{2}$ is the irreducible vertex function. To lowest order in the expansion (33) the denominator in Eq. (44) vanishes, which causes $W$ to diverge. To eliminate this divergence also the second term in Eq. (33) should be taken into account. We only evaluated $W$ for $\bar{\gamma}=0$ and $N_{T}=N$. Under these conditions the self-energy is purely imaginary, which simplifies the calculations and we obtain

$$
\left\langle\left|t_{\vec{k}_{\|}, \vec{k}_{\|}^{\prime}}\right|^{2}\right\rangle=\frac{\langle g\rangle k_{\perp} k_{\perp}^{\prime}}{\left(\sum_{\vec{k}_{\|}^{\prime \prime}} k_{\perp}^{\prime \prime}\right)^{2}}
$$

The configurationally averaged reflection probabilities can be calculated from the continuity of the wave function (11):
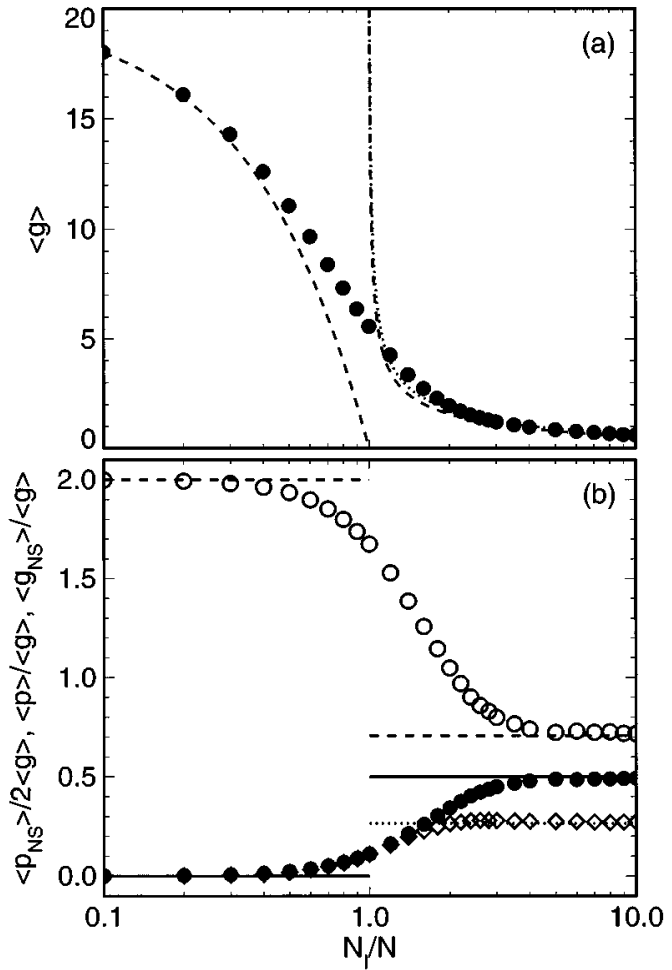

FIG. 11. Dependence of (a) the average conductance $\langle g\rangle$ and (b) the ratios $\langle p\rangle /\langle g\rangle,\left\langle g_{\mathrm{NS}}\right\rangle /\langle g\rangle$, and $\left\langle p_{\mathrm{NS}}\right\rangle / 2\langle g\rangle$ on the number of scatterers $N_{I}$. The numerical calculations were carried out using $N=20, N_{T}=20, \tilde{\gamma}=10.0$, and $\bar{\gamma}=0.0$. Configurational averaging was done using 10000 realizations of the disorder. For explanation of the symbols and the lines for $N_{I}>N$, see Fig. 7. The lines for $N_{I}<N$ are the results obtained in Ref. 15 for a low density of strong scatterers.

$$
\left\langle\left|r_{\vec{k}_{\|}, \vec{k}_{\|}^{\prime}}\right|^{2}\right\rangle=\left(1-\frac{2\langle g\rangle k_{\perp}}{\sum_{\vec{k}_{\|}^{\prime \prime}} k_{\perp}^{\prime \prime}}\right) \delta_{\vec{k}_{\|}, \vec{k}_{\|}^{\prime}}+\frac{\langle g\rangle k_{\perp} k_{\perp}^{\prime}}{\left(\sum_{\vec{k}_{\|}^{\prime \prime}} k_{\perp}^{\prime \prime}\right)^{2}} .
$$

In the SCBA the conductance calculated directly from the two-particle Green function is identical to the conductance calculated using the one-particle Green function and the Ward identity (20). More generally, the relation $\left\langle\mathbf{t}_{p} \mathbf{t}_{p}^{\dagger}\right\rangle$ $=\left(\left\langle\mathbf{t}_{p}\right\rangle+\left\langle\mathbf{t}_{p}^{\dagger}\right\rangle\right) / 2$ is satisfied in the SCBA. This means that, at

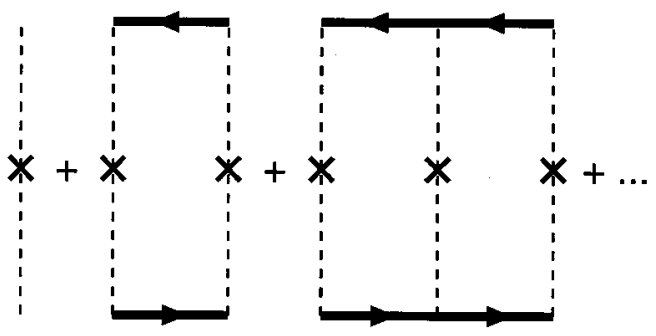

FIG. 12. Ladder diagrams that are taken into account in the calculation of the reducible vertex function matrix. The thick lines with a right pointing arrow represent a perturbed electron Green function; those with a left pointing arrow represent a perturbed hole Green function. 

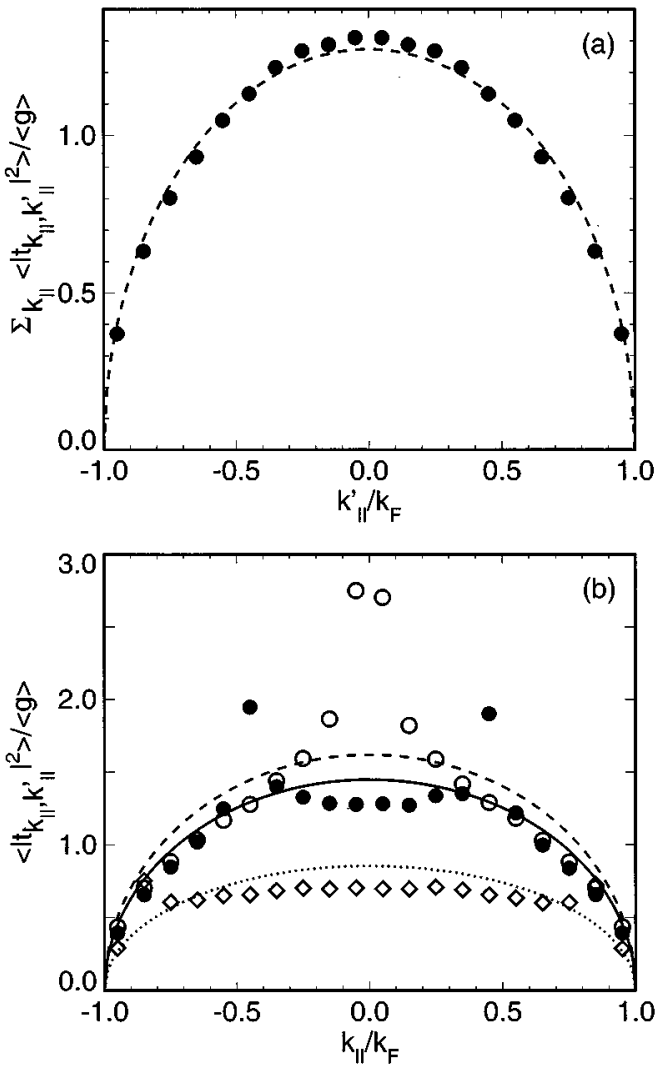

FIG. 13. Dependence of the (integrated) average transmission probabilities on the incoming or outgoing wave vectors. The numerical calculations were carried out using $N=20, N_{T}=20, N_{I}$ $=2000, \tilde{\gamma}=10.0$, and $\bar{\gamma}=0.0$. Configurational averaging was done using 10000 realizations of the disorder. (a) The numerically calculated transmission probabilities (filled circles) integrated over the outgoing states as a function of the incoming wave vector $k_{\|}^{\prime}$ compared with the SCBA result (dashed line). (b) The numerically calculated transmission probabilities as a function of the outgoing state $k_{\|}$for incoming state $k_{\|}^{\prime}$ equal to $0.05 k_{F}$ (open circles), $0.45 k_{F}$ (filled circles), and $0.85 k_{F}$ (diamonds) compared with the SCBA results given by the dashed, solid, and dotted lines, respectively.

least under the restrictions $\bar{\gamma}=0$ and $N_{T}=N$, the SCBA is consistent with the Ward identity (20), which is a necessary condition for any physically meaningful approximation. Note that the divergence of $W$ to lowest order in the expansion (33) is essential for current conservation.

The irreducible vertex function in the CPA consists of all noncrossed diagrams. A calculation similar to that in the SCBA shows that in the CPA the denominator in Eq. (44) does not vanish when $\bar{\gamma} \neq 0$. This implies that in the strong scattering regime the CPA does not conserve current for $\bar{\gamma}$ $\neq 0$ which we expect to be related to the qualitative deviation from the exact numerical results for $\bar{\gamma}>0$ in Fig. 10. At least for $\bar{\gamma} \neq 0$, the CPA should be applied to the strong scattering limit with care.

We compare the analytical results in the SCBA for $\bar{\gamma}$ $=0$ and $N_{T}=N$ with exact numerical calculations that are limited to $d=2$ and $\gamma_{\alpha}= \pm \gamma$. The transmission probabilities are obtained straightforwardly from Eq. (16). Figure 13(a) shows the transmission probability averaged over 10000 re- alizations of the disorder, normalized by $\langle g\rangle$, and summed over all outgoing states as a function of the incoming state. The numerical results agree well with the SCBA calculation for this integrated quantity. The dependence of the average transmission probability as a function of the outgoing state for one specific incoming state, however, is not well described by the SCBA result, as illustrated by Fig. 13(b). The enhanced probabilities for $k_{\|}=k_{\|}^{\prime}$ and $k_{\|}=-k_{\|}^{\prime}$ are quite striking. By performing a similar calculation for a smaller value of $\langle g\rangle$ we checked that the peak for $k_{\|}=k_{\|}^{\prime}$ is not due to a small ballistic component. From the relation (11) between $\mathbf{t}$ and $\mathbf{r}$ it follows that the nondiagonal terms of the reflection probability matrix are equal to the corresponding transmission probabilities and therefore similar peaks occur in the reflection probabilities. Since in the calculation of Fig. $13 N_{I}=100 N$, the deviation from the SCBA result is not caused by higher order noncrossed diagrams, which can be disregarded for $N_{I} \gg N$. Even in the CPA, in which all noncrossed diagrams are included, all elements in the reducible vertex function matrix $\mathbf{W}$ are identical, ${ }^{18}$ which implies that the angular dependence of transmission and reflection probabilities are also in this case given by Eqs. (45) and (46), respectively. This leaves the crossed diagrams as the origin of the peaks in the numerical calculations of Fig. 13(b). The enhanced reflection probabilities for $k_{\|}=-k_{\|}^{\prime}$ are analogous to the enhanced backscattering peak in bulk conductors, which is due to an interference effect that is described by crossed diagrams. ${ }^{21}$ A deeper study of the crossed diagrams is again beyond the scope of the present paper.

\section{DISCUSSION}

In this section we compare the results obtained for phasecoherent electrical transport through dirty interfaces with the well-known properties of disordered bulk conductors and discuss the similarities and the differences. Both distribution functions (4) and (35) for disordered bulk conductors and dirty interfaces, respectively, are bimodal. For dirty interfaces there is, however, relatively more weight for small $T_{n}$. This is reflected in the expectation values for the physical quantities. The ratio $\langle p\rangle /\langle g\rangle$, for example, equals $1 / 2$ for dirty interfaces compared to $1 / 3$ for disordered bulk conductors. In the case of a bulk disordered normal metal in contact with a superconductor $\left\langle g_{\mathrm{NS}}\right\rangle$ equals the conductance in the normal state and the ratio $\left\langle p_{\mathrm{NS}}\right\rangle /\left\langle g_{\mathrm{NS}}\right\rangle=2 / 3$, which is twice the normal state result. In contrast, for a dirty interface in a normal-metal in series with a superconductor $\left\langle g_{\mathrm{NS}}\right\rangle$ and $\langle g\rangle$ are no longer equal $\left(\left\langle g_{\mathrm{NS}}\right\rangle=\frac{1}{2} \sqrt{2}\langle g\rangle\right)$ and the ratio $\left\langle p_{\mathrm{NS}}\right\rangle /\left\langle g_{\mathrm{NS}}\right\rangle=3 / 4$, less than twice the normal-state result. These differences should be observable experimentally.

For disordered bulk conductors, the bimodal distribution function (4) has been related to the occurrence of universal conductance fluctuations. ${ }^{22}$ We investigate the conductance fluctuations of dirty interfaces by performing a straightforward numerical calculation of the variance of the conductance $\operatorname{Var}(g) \equiv\left\langle g^{2}\right\rangle-\langle g\rangle^{2}$. Figure 14 shows the dependences of $\langle g\rangle, \operatorname{Var}(g)$, and the ratios characterizing the distribution function on the number of conduction channels. As expected, the average conductance is proportional to $N$ and the distribution function is universal. The variance of the conductance is not independent of the number of conduction 


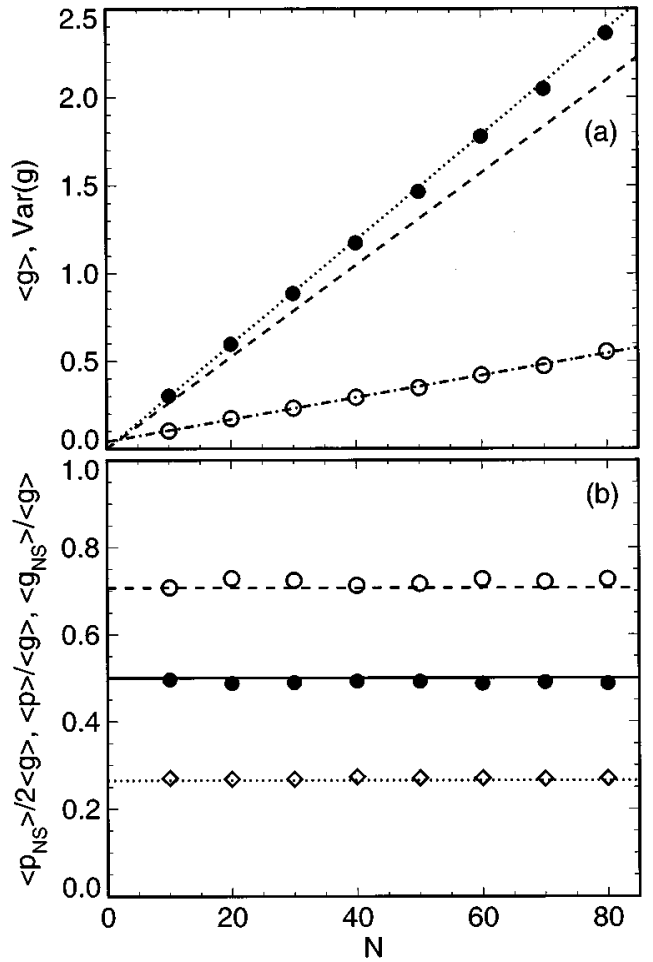

FIG. 14. Dependence of (a) the average conductance $\langle g\rangle$, the variance $\operatorname{Var}(g)$ and (b) the ratios $\langle p\rangle /\langle g\rangle,\left\langle g_{\mathrm{NS}}\right\rangle /\langle g\rangle$, and $\left\langle p_{\mathrm{NS}}\right\rangle / 2\langle g\rangle$ on the number of conduction channels $N$. The numerical calculations were carried out using $N_{T}=N, N_{I}=10 N, \tilde{\gamma}=10.0$, and $\bar{\gamma}=0.0$. Configurational averaging was done using 10000 realizations of the disorder. (a) The numerically calculated values of $\langle g\rangle$ (filled circles) compared with the CPA result (dashed line) and with the CPA result multiplied by a factor 1.14 (dotted line) as well as the numerically calculated values of $\operatorname{Var}(g)$ (open circles). The dash-dotted line is a linear fit $\operatorname{Var}(g) \approx 0.041+0.0063 N$. (b) The numerically calculated values of the ratios $\langle p\rangle /\langle g\rangle$ (filled circles), $\left\langle g_{\mathrm{NS}}\right\rangle /\langle g\rangle$ (open circles), and $\left\langle p_{\mathrm{NS}}\right\rangle / 2\langle g\rangle$ (diamonds) compared with their universal values given by the solid, dashed, and dotted lines, respectively.

channels, i.e., the conductance fluctuations are not universal. Instead, $\operatorname{Var}(g)$ increases linearly with $N$, as expected from classical arguments. In terms of the two-particle diagrams that describe the conductance fluctuations ${ }^{15}$ the nonuniversal behavior is due to the absence of a divergence in the ladder summations. Such a divergence occurs in Eq. (44) in the calculation of the transmission probabilities but is absent for the fluctuation diagrams. In the theory of the conductance fluctuations in bulk conductors the divergence in the ladder summation is the origin of the universality. ${ }^{2}$ The fluctuations are also nonuniversal close to the ballistic regime $(g \lesssim N){ }^{23}$

Because $P(T)$ for a dirty interface does not depend on the microscopic details, its calculation seems well suited for methods of random matrix theory. ${ }^{12}$ Continuity of the wave functions on both sides of the scattering region gives rise to the additional constraint $\mathbf{t}=\mathbf{I}+\mathbf{r}$ on the space of allowed scattering matrices. By combining this with the constraint that arises from current conservation ${ }^{12}$ we find that the transmission amplitude matrix for propagating states can always be parametrized as $\mathbf{t}_{p}=[\mathbf{I}+i \hat{\Gamma}]^{-1}$ in which $\hat{\Gamma}$ is a Hermitian matrix. In our specific model for randomly distributed $\delta$ scat- terers $\hat{\Gamma}$ equals $\widetilde{\Gamma}$, but another model for the interface roughness may yield a different expression for $\hat{\Gamma}$. We expect that this will not change the results obtained in this paper qualitatively and that the constraint $\mathbf{t}=\mathbf{I}+\mathbf{r}$ is sufficient to describe the characteristic properties of interfaces compared to bulk systems. A study of transport through dirty interfaces using random matrix theory might further clarify the origin of the universal result for $P(T)$ and the nonuniversal result for the conductance fluctuations.

The distribution function (35) for a dirty interface is identical to $P(T)$ for two identical tunnel barriers in series separated by a distance that is much larger than $\lambda_{F},{ }^{24}$ despite the fact that the two physical systems are very different. At this moment we have no physical argument to explain this correspondence.

In the above analytical treatment there is no qualitative difference between the regimes $g>1$ and $g<1$, i.e., no localization transition is observed. This is confirmed by the numerical calculations of Fig. 14, where the conductance varies between 0.3 and 2.4. No changes are observed in the universal ratios or in the proportionality between $\langle g\rangle$ and $N$. The absence of localization follows from the well-behaved maximally crossed diagrams ${ }^{15}$ and can be understood from the fact that for an interface the region of scattering has no spatial extent in the transport direction so there is no space available in which the wave functions can localize. In other words, any incipient bound state is immediately destroyed by the strong coupling to the continuum states in the leads on either side of the interface.

The transmission and reflection probabilities calculated in Sec. VII are quite different from the scattering probabilities for disordered bulk conductors. ${ }^{25}$ For dirty interfaces most of the reflection is specular in contrast to the smooth distribution over all reflection directions for bulk systems. This almost complete specular reflection is a direct consequence of the continuity relation $\mathbf{t}=\mathbf{I}+\mathbf{r}$ from which it is clear that the diagonal terms of the reflection probability matrix are close to unity for small transmission. This result is relevant for the study of electrical transport in metallic multilayers in which the interfaces are sometimes modeled by thin regions of bulk material with high resistivities. ${ }^{26}$ Although this description is valid for weak scattering ${ }^{27,18}$ it is not correct in the strong scattering regime. Due to quantum interference effects, additional peaks occur in the transmission and reflection probabilities for $\vec{k}_{\|}=\vec{k}_{\|}^{\prime}$ and $\vec{k}_{\|}=-\vec{k}_{\|}^{\prime}$. Whereas the enhanced reflection for $\vec{k}_{\|}=-\vec{k}_{\|}^{\prime}$ is analogous to the well-known enhanced backscattering, the other peaks have no counterparts in the bulk. The peaks in reflection and transmission for $\vec{k}_{\|}=\vec{k}_{\|}^{\prime}$ will be difficult to resolve experimentally because of the large specular reflection and the nonzero ballistic transmission, respectively. The enhanced transmission for $\vec{k}_{\|}=$ $-\vec{k}_{\|}^{\prime}$, on the other hand, might be observable experimentally. This peak is intimately related to the enhanced backscattering via the continuity relation (11).

In the analytical calculations only the noncrossed diagrams are evaluated explicitly, whereas the crossed diagrams that correspond to quantum interference are disregarded. Although in the strong scattering regime there is no a priori justification for such a mean-field approach, most of the re- 
sults obtained agree quite well with the numerical calculations. The suppression of the shot-noise power, for example, can be understood on the basis of a CPA or SCBA calculation. Also in disordered bulk conductors quantum interference effects are not required to describe the suppression of the shot-noise power. ${ }^{28}$

In summary, starting from the Schrödinger equation, we studied the phase-coherent electrical transport through dirty interfaces. We have shown that $P(T)$ for a dirty interface is universal but differs from $P(T)$ for disordered bulk conductors. Dirty interfaces belong to a universality class different from diffusive bulk conductors. In addition, we have shown that the conductance fluctuations are nonuniversal, the localization transition is absent, and the transmission and reflection probabilities are different from the bulk. It remains a challenge to test these results theoretically by random matrix theory and experimentally by transport studies of intentionally disordered metallic point contacts and wide quantum wires.

Note added in proof. Carlo Beenakker pointed out to us that for bulk diffusive conductors much wider than long, the mean-squared fluctuations are also proportional to the number of conducting channels $N$. We note that the slope of the linear dependence is not universal for the dirty interfaces.

\section{ACKNOWLEDGMENTS}

We thank Marc de Jong, Paul Kelly, and Yuli Nazarov for discussions. This work is part of the research program of the Stichting voor Fundamenteel Onderzoek der Materie, which is financially supported by the Nederlandse Organisatie voor Wetenschappelijk Onderzoek. We acknowledge benefits from the TMR Research Network on Interface Magnetism under Contract No. FMRX-CT96-0089 (DG12-MIHT).

\section{APPENDIX: DERIVATION OF EQ. (23)}

In this appendix the derivation of Eq. (23) is presented. In Eq. (22) the distribution function was written in terms of a power series in the transmission matrix. The first step in the derivation is to express higher-order moments in terms of a single transmission matrix. Using the Ward identities (20) and (21) we find for the second-order moment

$$
\left(\mathbf{t}_{p} \mathbf{t}_{p}^{\dagger}\right)^{2}=\left(\frac{1}{2} m \frac{\partial}{\partial m}+1\right) \mathbf{t}_{p} \mathbf{t}_{p}^{\dagger}=\left(\eta \frac{\partial}{\partial \eta}+1\right) \mathbf{t}_{p} \mathbf{t}_{p}^{\dagger},
$$

with $\eta=m^{2}$. Using Eq. (A1) we obtain a recursion relation for the higher-order moments:

$$
\left(\mathbf{t}_{p} \mathbf{t}_{p}^{\dagger}\right)^{n+1}=\frac{1}{n}\left(\eta \frac{\partial}{\partial \eta}+n\right)\left(\mathbf{t}_{p} \mathbf{t}_{p}^{\dagger}\right)^{n}
$$

By induction we then find from Eqs. (A1) and (A2) that

$$
\begin{aligned}
\left(\mathbf{t}_{p} \mathbf{t}_{p}^{\dagger}\right)^{n+1} & =\frac{1}{n !}\left[\prod_{m=1}^{n}\left(\eta \frac{\partial}{\partial \eta}+m\right)\right] \mathbf{t}_{p} \mathbf{t}_{p}^{\dagger} \\
& \equiv \hat{f}_{n}\left(\eta \frac{\partial}{\partial \eta}\right) \mathbf{t}_{p} \mathbf{t}_{p}^{\dagger}
\end{aligned}
$$

where the operator $\hat{f}_{n}(\hat{x})$ is a polynomial of order $n$ in the operator $\hat{x}$. Substituting Eq. (A3) into Eq. (22) and exchanging the orders of differentiation, configurational averaging, and taking the trace, $P(T)$ can be written as

$$
P(T)=\int \frac{d q}{2 \pi} e^{-i q T}\left[N+\sum_{n=1}^{\infty} \frac{(i q)^{n}}{n !} \hat{f}_{n-1}\left(\eta \frac{\partial}{\partial \eta}\right)\langle g(\eta)\rangle\right] .
$$

The calculation of the distribution function is thus reduced to the calculation of the average conductance and its dependence on $\eta$.

For explicit calculations Eq. (A4) is cumbersome. To derive a more convenient expression we divide the distribution function is three parts. The first part $P_{1}(T)$ contains only the first term inside the square brackets in Eq. (A4) and can easily be evaluated as

$$
P_{1}(T) \equiv \int \frac{d q}{2 \pi} e^{-i q T} N=N \delta(T)
$$

The second part $P_{2}(T)$ is the contribution to the distribution function that comes from the integrable part (denoted by the horizontal line) of the configurationally averaged conductance:

$$
P_{2}(T) \equiv \int \frac{d q}{2 \pi} e^{-i q T} \sum_{n=1}^{\infty} \frac{(i q)^{n}}{n !} \hat{f}_{n-1}\left(\eta \frac{\partial}{\partial \eta}\right) \overline{\langle g(\eta)\rangle}
$$

A simplified expression for $P_{2}(T)$ can be derived using a Kramers-Kronig relation for the integrable part of $\langle g(\eta)\rangle$ in the variable $\eta$

$$
\overline{\langle g(\eta)\rangle}=\operatorname{Re} \overline{\langle g(\eta)\rangle}=\mathrm{P} \int_{-\infty}^{\infty} \frac{d y}{-\pi} \frac{\operatorname{Im} \overline{\langle g(y)\rangle}}{y-\eta},
$$

where $\overline{\langle g(y)\rangle}$ is the analytical continuation of the integrable part of $\langle g(\eta)\rangle$ in the lower half complex plane and P denotes the principal part. We made use of the fact that the conductance is real for $\eta \geqslant 0 . P_{2}(T)$ can be calculated by substituting Eq. (A7) into Eq. (A6). To evaluate the resulting expression we first note that

$$
\hat{f}_{n-1}\left(\eta \frac{\partial}{\partial \eta}\right) \eta^{M}=\eta^{M} f_{n-1}(M)
$$

with

$$
f_{n-1}(M)=\left(\begin{array}{c}
M+n \\
n
\end{array}\right) .
$$

The operators that act on powers of $\eta$ can thus be replaced by numbers. Using this property we obtain

$$
\begin{aligned}
\hat{f}_{n-1}\left(\eta \frac{\partial}{\partial \eta}\right) \frac{1}{y-\eta} & =\frac{1}{y} \hat{f}_{n-1}\left(\eta \frac{\partial}{\partial \eta}\right) \sum_{M=0}^{\infty}\left(\frac{\eta}{y}\right)^{M} \\
& =\frac{1}{y} \sum_{M=0}^{\infty} f_{n-1}(M)\left(\frac{\eta}{y}\right)^{M}=\frac{1}{y}\left(\frac{y}{y-\eta}\right)^{n} .
\end{aligned}
$$


Substituting Eq. (A7) into Eq. (A6) and exchanging the order of differentiation and integration over $y$, Eq. (A6) can be simplified using Eq. (A10) to

$$
P_{2}(T)=\int \frac{d q}{2 \pi} e^{-i q T} \sum_{n=1}^{\infty} \frac{(i q)^{n}}{n !} P \int \frac{d y}{-\pi} \frac{\operatorname{Im} \overline{\langle g(y)\rangle}}{y}\left(\frac{y}{y-\eta}\right)^{n} .
$$

Using the fact that

$$
\operatorname{Im} \int \frac{d q}{2 \pi} e^{-i q T} \frac{(i q)^{n}}{n !}=0
$$

for all values of $n$ and separating the $n=0$ term we find

$$
\begin{aligned}
P_{2}(T) & =-\overline{\langle g(0)\rangle} \delta(T)+\operatorname{ImP} \int \frac{d y}{-\pi} \frac{\overline{\langle g(y)\rangle}}{y} \delta\left(T-\frac{y}{y-\eta}\right) \\
& =-\overline{\langle g(0)\rangle} \delta(T)+\frac{1}{\pi} \frac{1}{T(1-T)} \operatorname{Im} \overline{\left\langle g\left(\frac{\eta T}{T-1}-i 0^{+}\right)\right\rangle},
\end{aligned}
$$

where the positive infinitesimal $0^{+}$is added to indicate that the conductance should be calculated just below the real axis.

The last part $P_{3}(T)$ arises from the contributions of the nonintegrable parts of $\langle g(\eta)\rangle$, which should be treated separately. As an example we consider the contribution from $\langle g(\infty)\rangle$, which vanishes for dirty interfaces but remains finite in the limit of a small number of strong scatterers. ${ }^{15}$ Since $\langle g(\infty)\rangle$ is a constant not depending on $\eta$,

*Present and permanent address: Philips Research Laboratories, Prof. Holstlaan 4, 5656 AA Eindhoven, The Netherlands.

${ }^{1}$ For review articles see Mesoscopic Phenomena in Solids, edited by B. L. Altshuler, P. A. Lee, and R. A. Webb (North-Holland, Amsterdam, 1991)

${ }^{2}$ B. L. Altshuler, Pis'ma Zh. Éksp. Teor. Fiz. 41, 530 (1985) [ JETP Lett. 41, 648 (1985)]; P. A. Lee and A. D. Stone, Phys. Rev. Lett. 55, 1622 (1985).

${ }^{3}$ W. P. Pratt, Jr., S. -F. Lee, J. M. Slaughter, R. Loloee, P. A. Schroeder, and J. Bass, Phys. Rev. Lett. 66, 3060 (1991); M. A. M. Gijs, S. K. J. Lenczowski, and J. B. Giesbers, ibid. 70, 3343 (1993).

${ }^{4}$ For recent reviews see P. M. Levy, Solid State Phys. 47, 367 (1994); M. A. M. Gijs and G. E. W. Bauer, Adv. Phys. 46, 285 (1997).

${ }^{5}$ It is, however, still a subject of controversy whether the strong scattering at these interfaces comes from diffuse scattering due to interface roughness or from specular scattering due to mismatch of the band structures; see, e.g., K. M. Schep et al., Phys. Rev. B 56, 10805 (1997).

${ }^{6}$ K.-J. Friedland, T. Fujizawa, and S. Tarucha (unpublished).

${ }^{7}$ C. W. J. Beenakker, Phys. Rev. B 47, 15763 (1993).

${ }^{8}$ G. B. Lesovik, Pis'ma Zh. Éksp. Teor. Fiz. 49, 513 (1989) [ JETP Lett. 49, 592 (1989)]; M. Büttiker, Phys. Rev. Lett. 65, 2901 (1990)

${ }^{9}$ C. W. J. Beenakker, Phys. Rev. B 46, 12841 (1992).

${ }^{10}$ M. J. M. de Jong and C. W. J. Beenakker, Phys. Rev. B 49, 16070 (1994).

$$
\hat{f}_{n}\left(\eta \frac{\partial}{\partial \eta}\right)\langle g(\infty)\rangle=\langle g(\infty)\rangle
$$

and

$$
\begin{aligned}
P_{3}(T) & \equiv \int \frac{d q}{2 \pi} e^{-i q T} \sum_{n=1}^{\infty} \frac{(i q)^{n}}{n !} \hat{f}_{n-1}\left(\eta \frac{\partial}{\partial \eta}\right)\langle g(\infty)\rangle \\
& =-\langle g(\infty)\rangle \delta(T)+\langle g(\infty)\rangle \delta(T-1)
\end{aligned}
$$

The total distribution function is the sum of $P_{1}(T)$, $P_{2}(T)$, and $P_{3}(T)$. Note that the integrals over $T$ of both $P_{2}(T)$ and $P_{3}(T)$ vanish and that $P(T)$ is thus properly normalized. In Eq. (23) only $P_{1}(T)$ and $P_{2}(T)$ are taken into account since $\langle g(\infty)\rangle$ vanishes for dirty interfaces. When the conductance contains only integrable parts $\langle g(0)\rangle=N$ and the $\delta(T)$ terms arising from $P_{1}(T)$ and $P_{2}(T)$ cancel.

In principle full knowledge of $\langle g(\eta)\rangle$ for $\eta$ ranging from 0 to $\infty$ is required to calculate $P(T)$. Since our calculations for dirty interfaces are limited to $g \ll N$ they do not describe $\langle g(\eta)\rangle$ correctly for $\eta$ approaching zero, which causes incorrect behavior of the distribution function for small $T$. This results in the nonintegrable divergence at $T=0$ in Eq. (35) even though Eq. (23) is properly normalized. To normalize $P(T)$ correctly a cutoff at small $T$ was introduced in Eq. (35). This cutoff is irrelevant for the first- and higher-order moments of $T$. A similar cutoff occurs in Eq. (4) for disordered bulk conductors.
${ }^{11}$ O. N. Dorokhov, Solid State Commun. 51, 381 (1984).

${ }^{12}$ A. D. Stone, P. A. Mello, K. A. Muttalib, and J.-L. Pichard, in Mesoscopic Phenomena in Solids (Ref. 1), p. 369; C. W. J. Beenakker, Rev. Mod. Phys. 69, 731 (1997).

${ }^{13}$ Yu. V. Nazarov, Phys. Rev. Lett. 73, 134 (1994).

${ }^{14}$ C. W. J. Beenakker and M. Büttiker, Phys. Rev. B 46, 1889 (1992).

${ }^{15}$ G. E. W. Bauer, Phys. Rev. B 51, 16984 (1995).

${ }^{16}$ J. B. Pendry, A. MacKinnon, and P. J. Roberts, Proc. R. Soc. London, Ser. A 437, 67 (1992).

${ }^{17}$ K. M. Schep and G. E. W. Bauer, Phys. Rev. Lett. 78, 3015 (1997).

${ }^{18}$ A. Brataas and G. E. W. Bauer, Phys. Rev. B 49, 14684 (1994).

${ }^{19}$ P. F. Bagwell, Phys. Rev. B 41, 10354 (1990).

${ }^{20}$ D. S. Fisher and P. A. Lee, Phys. Rev. B 23, 6851 (1981).

${ }^{21}$ M. B. Hastings, A. D. Stone, and H. U. Baranger, Phys. Rev. B 50, 8230 (1994).

${ }^{22}$ Y. Imry, Europhys. Lett. 1, 249 (1986).

${ }^{23}$ Y. Asano and G. E. W. Bauer, Phys. Rev. B 54, 11602 (1996).

${ }^{24}$ J. A. Melsen and C. W. J. Beenakker, Physica B 203, 219 (1994). This correspondence was pointed out to us by M. J. M. de Jong.

${ }^{25}$ A. V. Tartakovski, Phys. Rev. B 52, 2704 (1995).

${ }^{26}$ B. L. Johnson and R. E. Camley, Phys. Rev. B 44, 9997 (1991).

${ }^{27}$ H. E. Camblong and P. M. Levy, Phys. Rev. Lett. 69, 2835 (1992).

${ }^{28}$ M. J. M. de Jong and C. W. J. Beenakker, Phys. Rev. B 51, 16867 (1995). 